\title{
The platelet membrane glycoprotein VI genetic polymorphism (rs1613662, 13254T>C) is not associated with the risk of coronary artery disease
}

\author{
Jian-Quan Luo ${ }^{1,2, *}$, Huan Ren ${ }^{3, *}$, Mou-Ze Liu ${ }^{1,2}$, Ping-Fei Fang ${ }^{1,2}$ and Da-Xiong Xiang ${ }^{1,2}$ \\ ${ }^{1}$ Department of Pharmacy, The Second Xiangya Hospital, Central South University, Changsha, Hunan 410011, P. R. China \\ ${ }^{2}$ Institute of Clinical Pharmacy, Central South University, Changsha, Hunan 410011, P. R. China \\ ${ }^{3}$ Department of Clinical Pharmacology, Xiangya Hospital, Central South University, Changsha 410008, P. R. China \\ *These authors contributed equally to this work \\ Correspondence to: Jian-Quan Luo, email: Ivojianquanxy@csu.edu.cn
}

Keywords: glycoprotein Vl; platelets; genetic association; polymorphism; coronary artery disease

Received: June 01, $2017 \quad$ Accepted: November 28, $2017 \quad$ Published: January 02, 2018

Copyright: Luo et al. This is an open-access article distributed under the terms of the Creative Commons Attribution License 3.0 (CC BY 3.0), which permits unrestricted use, distribution, and reproduction in any medium, provided the original author and source are credited.

\section{ABSTRACT}

The platelet membrane glycoprotein VI (GP VI), encoded by GP6 gene, is the essential platelet collagen receptor and medicates platelet activation, adhesion and aggregation. Numerous studies revealed that the GP6 genetic polymorphisms may be associated with the susceptibility of coronary artery disease (CAD). However, a clear consensus has not yet been established. To investigate the association between GP6 genetic polymorphisms and CAD, the databases Pubmed, Embase, Chinese National Knowledge Infrastructure (CNKI), and Wanfang were searched for related studies. The pooled odds ratios (ORs) and their corresponding $95 \%$ confidence intervals (CIs) were used to evaluate the strength of the association by using a random or fixed-effect model. Our analysis confirmed that there was no significant association between the GP6 13254T>C (Ser219Pro, rs1613662) genetic polymorphism and the risk of CAD under an allelic genetic model $(O R=1.00,95 \%$ CI $=0.79-1.27$; $P=0.988)$, a homozygous genetic model $(O R=1.14,95 \% C I=0.73-1.80 ; P=0.563)$, a heterozygous genetic model $(O R=1.12,95 \% C I=0.95-1.33 ; P=0.183)$, a recessive genetic model $(O R=1.11,95 \% C I=0.71-1.74 ; P=0.652)$. Sensitivity and subgroup analysis indicated the robustness of the results. No publication bias existed between studies. In conclusion, no significant associations between GP6 13254T>C genetic polymorphism and CAD risk were found in this meta-analysis. More large-scale studies on the association of other GP6 genetic polymorphisms and the risk of CAD are needed to be performed in the future.

\section{INTRODUCTION}

Cardiovascular diseases are the leading burden of morbidity and mortality worldwide, and coronary artery disease (CAD) accounts for the greatest proportion of cardiovascular diseases [1]. The major forms of CAD include: stable angina, unstable angina, acute coronary syndromes (ACS), myocardial infarction (MI). CAD has a complex pathophysiology determined by lifestyle, environmental and genetic factors [2]. The lifestyle- and environmental-related factors include cigarette smoking, salt intake, obesity, diet, hypertension, diabetes mellitus and other factors [3]. In the past decades, the genetic mechanisms underlying CAD predisposition are widely investigated by case-control association studies [4-11].

Patelet aggregation and thrombosis are involved in the pathogenesis of CAD. In platelets, membrane glycoproteins receptors play crucial roles in adhesion, activation and aggregation, a sequence of events resulting in thrombus formation. Glycoprotein VI (GPVI, GP6), a critical platelet membrane glycoprotein of the immunoglobulin (Ig) superfamily, is an essential receptor for collagen and 
medicates collagen-induced platelet aggregation and thrombus formation $[12,13]$. It is a 60 to $65 \mathrm{kDa}$ type I transmembrane platelet glycoprotein and contains two extracellular Ig-like domains. It forms a complex with the Fc receptor gamma-chain [14]. Upon blood vessel injury, the complex initiates the platelet activation signaling cascade through the exposed subendothelial collagen binding. The platelet-collagen interactions are associated with cardio- and cerebral-vascular diseases in pathologic conditions, and compounds targeting the GPVI-collagen axis have antiatherothrombotic potential $[15,16]$.

The GP6 gene is mapped on the chromosome 19q13.42 and contains 8 exons spanning over $23 \mathrm{kbp}$ [17]. In consideration of its vital roles in the platelet activation and aggregation, the GP6 gene is considered as an excellent candidate gene for association study in the CAD patients. A plethora of case-control studies have been conducted in order to identify the association between the GP6 13254T $>$ C (Ser219Pro, rs1613662) gene polymorphism and $\mathrm{CAD}$, but contradictory results have been published. Some studies have been reported that the GP6 13254C polymorphism was significantly associated with the susceptibility to CAD, and the GP6 13254C allele increased the risk of CAD [18]. On the contrary, Qin et al. and Yu et al. failed to observe a significant association between the GP6 13254C variant and CAD risk [19, 20]. He et al. also found a similar result in another population [21].

To address the current discordance in the previous findings, we sought to conduct a comprehensive metaanalysis to improve the estimation of association between the GP6 genetic polymorphisms and CAD in the population studies.

\section{RESULTS}

\section{Characteristics of eligible studies}

Our current meta-analysis was performed according to guidelines of the Meta-analysis of Observational Studies in Epidemiology (MOOSE) statement [22]. A total of 317 relevant papers were produced by our initial literature search, among which 11 studies met the inclusion criteria, including 2692 cases and 2838 controls. As is depicted in the flow diagram (Figure 1), 57 articles were removed because of duplicates and then 201 papers were excluded owing to the obvious irrelevance. The full texts of the remaining 59 articles were reviewed and 10 papers were eligible [18, 19, 21, 23-29]. The paper of Croft SA et al. [18] contained two studies conducted in Sheffield and Leicester, and the data in the paper were extracted seperately for each studies. Therefore, a total of 11 studies for the association between GP6 13254T $>\mathrm{C}$ polymorphism and CAD risk were included in our metaanalysis. The information collected from the selected studies was presented in the Table 1. Those countries were included in the meta-analysis as following: United Kingdom, Finland, Czech, Netherlands, USA, Iran and China.

\section{Results of meta-analysis}

There was no significant association between the GP6 13254T $>\mathrm{C}$ genetic polymorphism and CAD in the total population under an allelic genetic model $(\mathrm{OR}=1.00,95 \% \mathrm{CI}=0.79-1.27 ; P=0.988$, Table 2 and Figure 2), a homozygous genetic model (OR $=1.14$, $95 \% \mathrm{CI}=0.73-1.80 ; P=0.563)$, a heterozygous genetic model $(\mathrm{OR}=1.12,95 \% \mathrm{CI}=0.95-1.33 ; P=0.183)$, a recessive genetic model $(\mathrm{OR}=1.11,95 \% \mathrm{CI}=0.71-1.74$; $P=0.652)$. However, significant association between GP6 13254T $>$ C genetic polymorphism and CAD was found under a dominant genetic model $(\mathrm{OR}=1.17$, $95 \% \mathrm{CI}=1.01-1.37 ; P=0.041$, Table 2 and Figure 3 )

In the subgroup analysis, there was no significant association in the Chinese population under the allelic, heterozygous and dominant genetic models $(P>0.05$, Table 2). Owing to no individuals carrying the $\mathrm{CC}$ genotype, meta-analysis could not be made in the Chinese population under the recessive and homozygous genetic models. Additionally, no significant association was also observed in the non-Chinese population under all of the genetic models $(P>0.05)$ including the dominant genetic model $(\mathrm{OR}=1.17,95 \% \mathrm{CI}=0.997-1.37 ; P=0.054$, Table 2 and Figure 3).

\section{Sources of heterogeneity}

No significant heterogeneity in the overall population was found under all of the genetic models $\left(P_{\text {heterogeneity }}>0.05\right.$, $I^{2}<50 \%$ ) except under the allelic genetic model $\left(P_{\text {heterogeneity }}=0.01, P^{2}=59.9 \%\right)$. Subgroup analysis also showed that heterogeneity was only detected in the non-Chinese subgroup (minor allele frequency, MAF $>0.05$ ) under the allelic genetic model $\left(P_{\text {heterogeneity }}=0.002, I^{2}=76.8 \%\right)$. Therefore, ethnicity was the main confounding factor that could explain the heterogeneity between studies.

\section{Sensitivity analysis}

The influence of individual study on the pooled ORs and $95 \%$ CIs was evaluated by excluding one single study each time. The corresponding combined ORs and 95\% CIs were not significantly altered in the allelic (Figure 4A), recessive (Figure 4B) and homozygous (Figure 4C) genetic models, suggesting a high stability of our meta-analysis results. However, under a dominant model, the significant association $(\mathrm{OR}=1.17,95 \% \mathrm{CI}=1.01-1.37 ; P=0.041)$ was lost if the study by Croft(Sheffield) et al. [18], Ollikainen et al. [23], Motovska et al. [25], Kazemi et al. [28], He et al. [21], was removed (Table 3, Figure 4D). In addition, the omission of study by Croft(Leicester) et al. 
[18] led to a significant association under a heterozygous genetic model (Table 3, Figure 4E).

\section{Publication bias}

We performed the Egger's test and Begg's funnel plot to assess the publication bias of the included articles. The $p$-values for Egger's test and Begg's test were summarized in Table 4, which demonstrated that there was no publication bias of the current meta-analysis in all genetic models. And the shape of funnel plot by using allelic genetic model was consistent with these results (Figure 5).

\section{DISCUSSION}

The pivotal role of GP6 in platelet activation and aggregation raises the question as to whether the GP6 genetic polymorphisms contribute to the susceptibility of CAD. Several GP6 genetic polymorphisms were investigated in the pathogenesis of CAD. To enhance the statistical power, we conducted the present meta-analysis by using the previously published data. The reported genetic polymorphisms include T13254C, A19871G, A21908G, A22630T, C22644A and so on [18]. After the GP6 13254T >C polymorphism was firstly reported to confer an increased risk of $\mathrm{MI}$ in the UK, Takagi et.al found that another GP6 genetic polymorphism (C645213T) could also affect the occurrence of myocardial infarction in a Japanese population [30]. With respect to the GP6 13254T >C variant, it is quite paradoxical that the results range from association as a protective factor, to no association, to association as a positive risk factor. Owing to the limited studies on other GP6 genetic polymorphisms, meta-analysis was only conducted in the studies of GP6 13254T >C polymorphism.

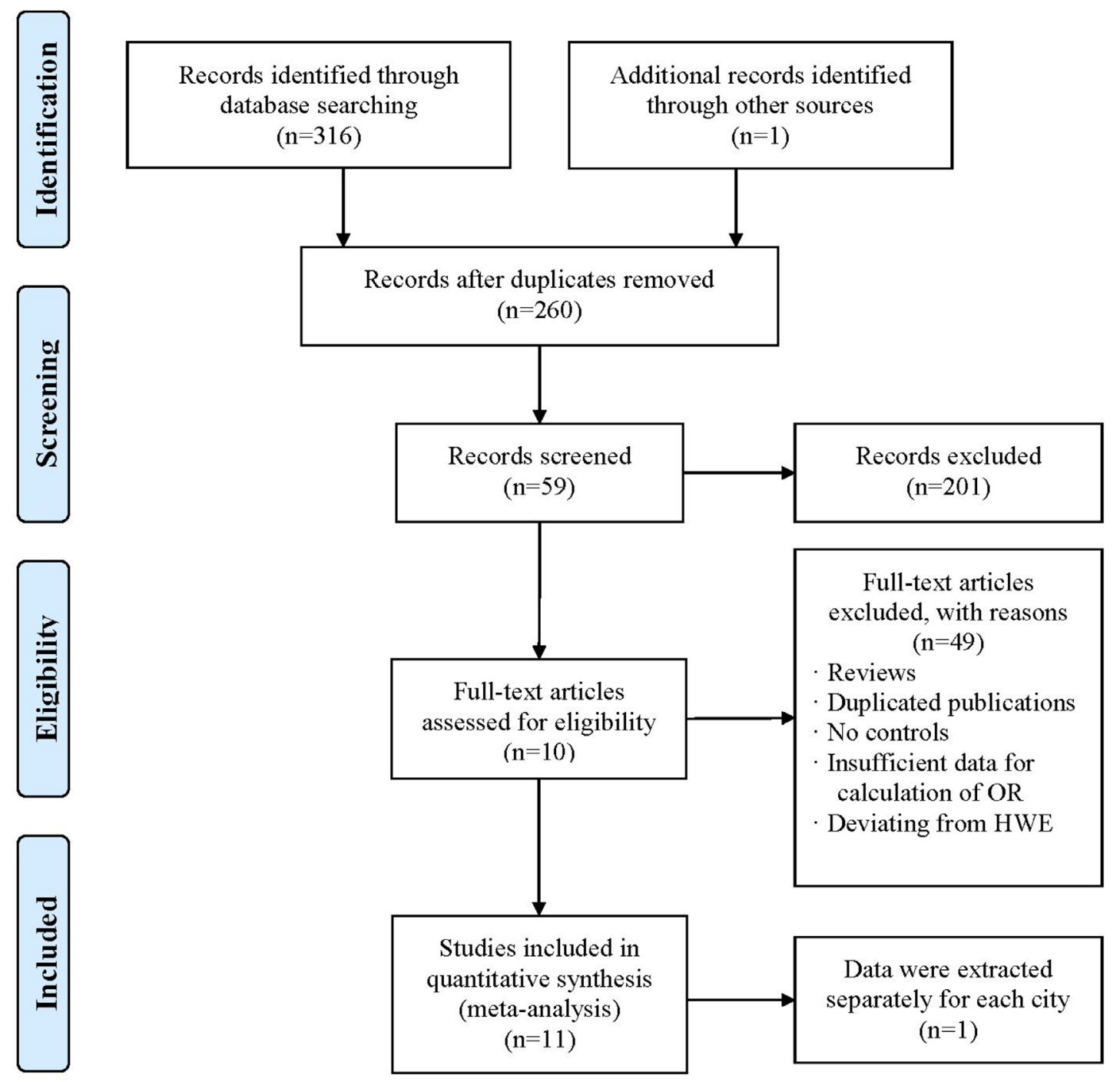

Figure 1: Flow diagram of the search strategy and study selection The terms " $n$ " in the boxes represent the number of corresponding studies. 
Table 1: Characteristics of the included studies of the association between the GP6 13254T $>$ C genetic polymorphism and coronary artery disease

\begin{tabular}{|c|c|c|c|c|c|c|c|c|c|c|c|c|c|c|c|c|c|c|}
\hline \multirow{2}{*}{$\begin{array}{l}\text { First } \\
\text { author }\end{array}$} & \multirow{2}{*}{ Year } & \multirow{2}{*}{ Region } & \multicolumn{2}{|c|}{ Age (years old) } & \multirow{2}{*}{$\begin{array}{c}\text { Genotyping } \\
\text { method }\end{array}$} & \multirow{2}{*}{$\begin{array}{c}\text { Sample size } \\
\text { (case/control) }\end{array}$} & \multicolumn{5}{|c|}{ Case } & \multicolumn{5}{|c|}{ Control } & \multirow{2}{*}{$\begin{array}{c}\text { MAF } \\
\text { (control) }\end{array}$} & \multirow{2}{*}{$\begin{array}{l}\text { Diagnostic criteria } \\
\text { (defnition) }\end{array}$} \\
\hline & & & Case & Control & & & TT & CT & $\mathrm{CC}$ & $\mathrm{T}$ & C & TT & CT & $\mathrm{CC}$ & $\mathrm{T}$ & $\mathrm{C}$ & & \\
\hline Croft & 2001 & UK (Sheffield) & $61.9 \pm 9.2$ & $61.1 \pm 9.1$ & PCR-RFLP & $289 / 292$ & 189 & 89 & 11 & 467 & 111 & 213 & 76 & 3 & 502 & 82 & 0.140 & WHO(MI) \\
\hline Croft & 2001 & UK (Leicester) & $61.5 \pm 9.3$ & $54.4 \pm 11.8$ & PCR-RFLP & $236 / 182$ & 166 & 60 & 10 & 392 & 80 & 118 & 57 & 7 & 293 & 71 & 0.195 & WHO(MI) \\
\hline Ollikainen & 2004 & Finland & NA & NA & PCR-RFLP & $67 / 250$ & 50 & NA & NA & NA & NA & 200 & 48 & 2 & 448 & 52 & 0.104 & NA(MI) \\
\hline Kou & 2004 & China & $60.0 \pm 6.3$ & $58.8 \pm 7.3$ & PCR-RFLP & $121 / 154$ & 112 & 9 & 0 & 233 & 9 & 145 & 9 & 0 & 299 & 9 & 0.029 & WHO(CAD) \\
\hline Qin & 2005 & China & $59.8 \pm 8.9$ & $57.2 \pm 9.6$ & PCR-RFLP & $179 / 164$ & 170 & 9 & 0 & 349 & 9 & 155 & 9 & 0 & 319 & 9 & 0.027 & WHO(ACS) \\
\hline Motovska & 2010 & Czech & $47.8 \pm 6.1$ & $63.66 \pm 9.47$ & PCR-RFLP & $105 / 137$ & 72 & 30 & 3 & 174 & 36 & 105 & NA & NA & NA & NA & NA & $\mathrm{ACC} / \mathrm{ESC}(\mathrm{MI})$ \\
\hline Snoep & 2010 & Netherlands & $57(49-64)$ & $59(50-66)$ & Taqman & $547 / 646$ & 366 & 163 & 16 & 895 & 195 & 446 & 176 & 21 & 1068 & 218 & 0.170 & NA(MI) \\
\hline Shaffer & 2011 & USA & NA & NA & Taqman & $652 / 625$ & NA & NA & NA & 1131 & 173 & NA & NA & NA & 1024 & 226 & 0.181 & WHO(MI) \\
\hline Kazemi & 2012 & Iran & $46.3 \pm 5.2$ & $44.7 \pm 6.8$ & PCR-RFLP & $100 / 100$ & 62 & 34 & 4 & 158 & 42 & 67 & 26 & 7 & 160 & 40 & 0.200 & $\mathrm{ACC} / \mathrm{ESC}(\mathrm{MI})$ \\
\hline Sun & 2012 & China & $76.0 \pm 7.5$ & $71.72 \pm 8.3$ & Sequenom & $246 / 185$ & 240 & 6 & 0 & 486 & 6 & 180 & 5 & 0 & 365 & 5 & 0.014 & $\mathrm{NA}(\mathrm{CAD})$ \\
\hline $\mathrm{He}$ & 2014 & China & $66(45-78)$ & $64(46-81)$ & ABI3730XL & $150 / 153$ & 146 & 4 & 0 & 296 & 4 & 153 & 0 & 0 & 306 & 0 & 0.000 & WHO(CAD) \\
\hline
\end{tabular}

available; PCR-RFLP, polymerase chain reaction-restriction fragment length polymorphism; UK, United Kingdom; USA, United States of America; WHO, World Health Organization.

Table 2: Summary of meta-analysis of association of GP6 13254T $>C$ genetic polymorphism and coronary artery disease

\begin{tabular}{|c|c|c|c|c|c|c|c|}
\hline Genetic model & $\begin{array}{c}\text { Pooled OR } \\
(95 \% \text { CI })\end{array}$ & $Z$-value & $P$-value & $\begin{array}{c}\text { Literature } \\
\text { number }\end{array}$ & Model & $\mathbf{P}_{\text {heterogeneity }}$ & $P^{2 \%}$ \\
\hline Allelic genetic model & $1.00(0.79-1.27)$ & 0.02 & 0.988 & 9 & $\mathrm{R}$ & 0.010 & $59.90 \%$ \\
\hline Chinese subgroup (MAF < 0.05) & $1.21(0.70-2.11)$ & 0.68 & 0.495 & 4 & $\mathrm{~F}$ & 0.482 & $0.00 \%$ \\
\hline Non-Chinese subgroup $(\mathrm{MAF}>0.05)$ & $0.98(0.75-1.29)$ & 0.13 & 0.897 & 5 & $\mathrm{R}$ & 0.002 & $76.80 \%$ \\
\hline Recessive genetic model & $1.11(0.71-1.74)$ & 0.45 & 0.652 & 4 & $\mathrm{~F}$ & 0.164 & $41.20 \%$ \\
\hline Chinese subgroup (MAF < 0.05) & NA & NA & NA & 0 & NA & NA & NA \\
\hline Non-Chinese subgroup (MAF $>0.05)$ & $1.11(0.71-1.74)$ & 0.45 & 0.652 & 4 & $\mathrm{~F}$ & 0.164 & $41.20 \%$ \\
\hline Dominant genetic model & $1.17(1.01-1.37)$ & 2.04 & 0.041 & 10 & $\mathrm{~F}$ & 0.366 & $8.30 \%$ \\
\hline Chinese subgroup (MAF < 0.05) & $1.22(0.70-2.13)$ & 0.69 & 0.49 & 4 & $\mathrm{~F}$ & 0.477 & $0.00 \%$ \\
\hline Non-Chinese subgroup (MAF $>0.05$ ) & $1.17(0.997-1.37)$ & 1.93 & 0.054 & 6 & $\mathrm{~F}$ & 0.194 & $32.20 \%$ \\
\hline Homozygous genetic model & $1.14(0.73-1.80)$ & 0.58 & 0.563 & 4 & $\mathrm{~F}$ & 0.163 & $41.50 \%$ \\
\hline Chinese subgroup (MAF < 0.05) & NA & NA & NA & 0 & NA & NA & NA \\
\hline Non-Chinese subgroup (MAF $>0.05)$ & $1.14(0.73-1.80)$ & 0.58 & 0.563 & 4 & $\mathrm{~F}$ & 0.163 & $41.50 \%$ \\
\hline Heterozygous genetic model & $1.12(0.95-1.33)$ & 1.33 & 0.183 & 8 & $\mathrm{~F}$ & 0.418 & $1.50 \%$ \\
\hline Chinese subgroup (MAF $<0.05$ ) & $1.22(0.70-2.13)$ & 0.69 & 0.490 & 4 & $\mathrm{~F}$ & 0.477 & $0.00 \%$ \\
\hline Non-Chinese subgroup (MAF $>0.05)$ & $1.11(0.93-1.33)$ & 1.18 & 0.239 & 4 & $\mathrm{~F}$ & 0.198 & $35.70 \%$ \\
\hline
\end{tabular}

CI, confidence interval; F, fixed-effects model; MAF, minor allele frequency; NA, not available; OR, odds ratio; R, random-effects model.

In our meta-analysis of the GP6 13254T $>\mathrm{C}$ genetic polymorphism, there was no significant association between and CAD in the total population under an allelic genetic model, a homozygous genetic model, a heterozygous genetic model, a recessive genetic model. The significant association between GP6 13254T $>\mathrm{C}$ genetic polymorphism and CAD was only found under a dominant genetic model. However, the significant association was not exited in the subgroups stratified by ethnicity. Therefore, the GP6 13254T >C polymorphism was not associated with CAD.

The mature GP6 protein consists of 319 amino acids and the GP6 gene $13254 \mathrm{~T}>\mathrm{C}$ (rs1613662) polymorphism is an amino acid substitution of serine 219 by proline (Ser219Pro) in the exon 5, which is a likely factor for the functional differences of two common GP6 haplotypes (GP6a and GP6b). The amino acid substitution was predicted to affect at least the local secondary/tertiary structure of the GPVI receptor. Previous studies indicated that the GP6 $13254 \mathrm{~T}>\mathrm{C}$ polymorphism played important roles in the expression of receptor, platelet activation and aggregation, signaling and fibrinogen binding [31, 32]. Trifiro et al. found that the GP6 $13254 \mathrm{~T}>\mathrm{C}$ polymorphism or the GP6a/GP6b haplotypes do not influence the GPVI ligand-binding affinity and expression levels [33]. This may partly explain why the association between the GP6 $13254 \mathrm{~T}>\mathrm{C}$ polymorphism and CAD risk was negative in our meta-analysis results. In addition, other genetic variants in the GP6 gene may also affect the expression of GP6 and the platelet function. The genomic structure GP6 is composed of 8 exons and 7 introns. Genetic variations in the regulatory region may alter the sequence of protein and lead to its functional effects. A recent study, conducted by Liu et al. [34], showed that GP6 rs1671153 and rs1654419 
genetic polymorphisms were significantly with the risk of CAD. The polymorphisms rs1671153 and rs1654419 were located in the intron 6 and intron 5 respectively, which may influence the splicing of GP6 and be involved in the regulation of mRNA stability. However, the mechanism needs to be investigated by functional validation of this particular SNP.

Based upon comparison of the MAF value in the included studies, we found there was a racial difference in the distribution of the GP6 13254T $>\mathrm{C}$ genetic polymorphism. The frequency of the GP6 13254C allele in Caucasian American and European population was nearly $20 \%[18,26,27]$, which is considerably different from the Chinese individuals (less than 5\%) [19,21] and the Japanese population (MAF $=2 \%$ ) [30]. In addition, The Iranian population (MAF $=20 \%$ ) [28] is genetically very similar to Caucasian American and European population individuals. Importantly, the difference in allele frequencies among ethnicities is also consistent with the data from the 1000 genomics database (https:// www.ncbi.nlm.nih.gov/variation/tools/1000genomes/).

Therefore, subgroup analysis stratified by the MAF value was conducted in our meta-analysis.

To the best of our knowledge, this is a comprehensive report with the largest sample size to determine the association between GP6 13254T $>$ C genetic polymorphism and the susceptibility of CAD by a metaanalysis. The major strength of this current study was lack of significant heterogeneity under all of the genetic models except under the allelic genetic model. The sources of between-study heterogeneity were explored by the subgroup analysis, and the results showed that ethnicity contributed to heterogeneity in the allelic genetic model. Sensitivity analysis also demonstrated that the omission of any individual study did not significantly change the pooled estimates of meta-analysis under the allelic, recessive and homozygous genetic models. Additionally, in the analysis of publication bias, the Begg's funnel plot

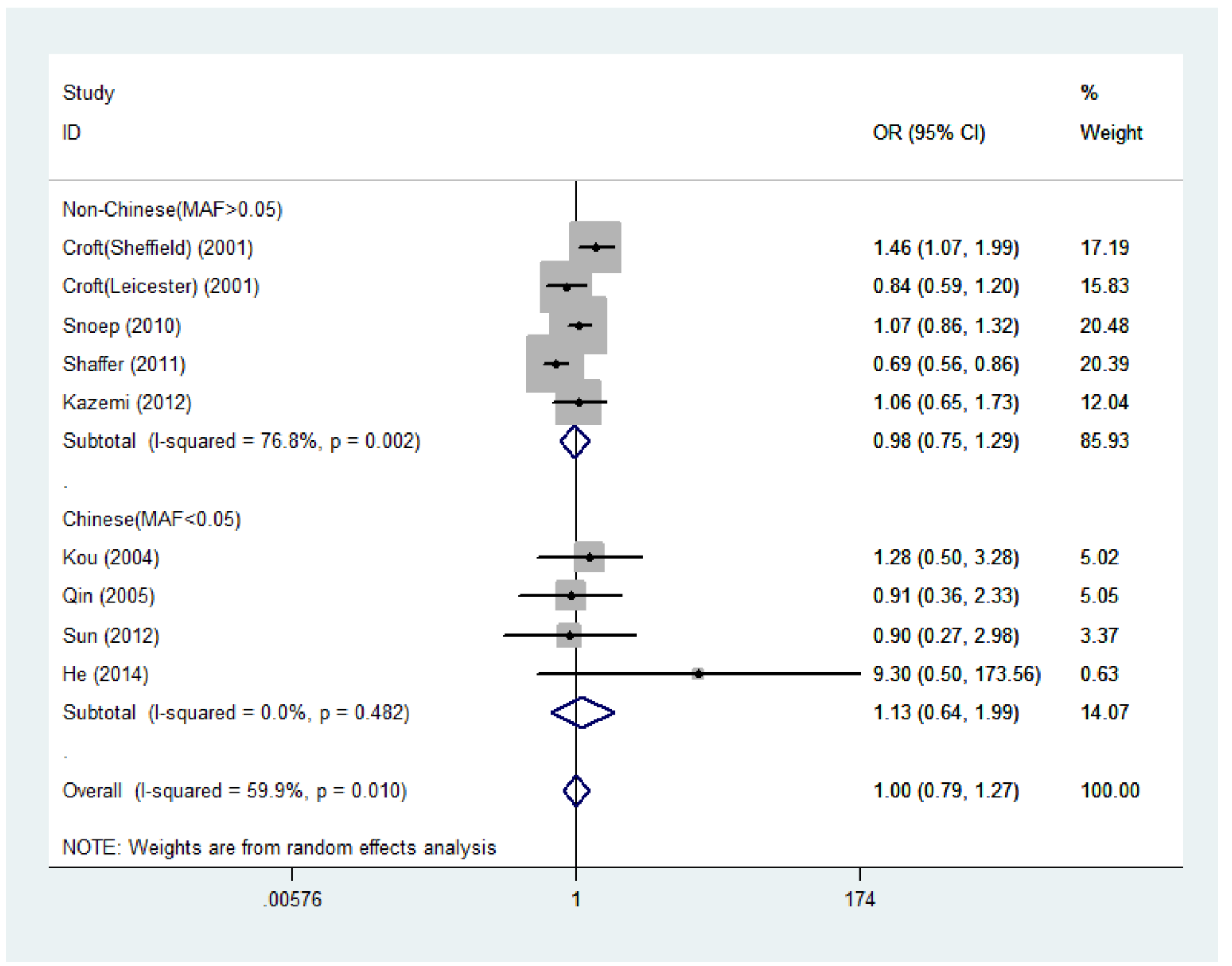

Figure 2: Forest plot for the allelic genetic model stratified by ethnicity (minor allele frequency of GP6 13254T $>$ C polymorphism) in studies with coronary artery disease patients. 
did not reflect remarkable asymmetry and the results of Egger's test were consistent in all genetic models. Therefore, all these analyses made our meta-analysis findings robust and reliable.

However, our meta-analysis is not without limitations. Firstly, since the common environmental risk factors (e.g. age, gender, obesity, dyslipidemia, hypertension, diabetes or smoking) of the CAD were not available in the included studies, we were unable to obtain these factors to adjust the meta-analysis. Thus, the results in our meta-analysis were based primarily on the crude ORs and its 95\% CIs. Secondly, because the included studies were all retrospective case-control association researches, some potential undetected bias may not be excluded. Thirdly, an interaction between hormone replacement therapy and GP6 gene on risk for CAD existed in the previous reports [27], but similar analysis weren't be performed in our article due to lack of the information in most studies. Fourthly, sensitivity analysis showed that the pooled results could be altered by some studies under the dominant and heterozygous model, and thus these need to be interpreted with caution. Last but not the least, owing to a polygenic disease like CAD, the effect of haplotypes or gene-gene interaction deserved to be investigated. Even though several GP6 genetic polymorphisms have been associated with CAD [30], the pooled results regarding other GP6 variants can't be available because of the limited studies.

In conclusion, this meta-analysis showed that the GP6 13254T $>\mathrm{C}$ (rs1613662) polymorphism was not significantly associated with $\mathrm{CAD}$ risk. Given the limitations mentioned above, more large-scale studies on the association of other GP6 genetic polymorphisms (e.g. rs1671153, rs1654419) and the risk of CAD deserved to be performed to further confirm our findings in the future.

\section{MATERIALS AND METHODS}

\section{Search strategy and selection criteria}

We performed a systematic published data search for the association studies between GP6 genetic

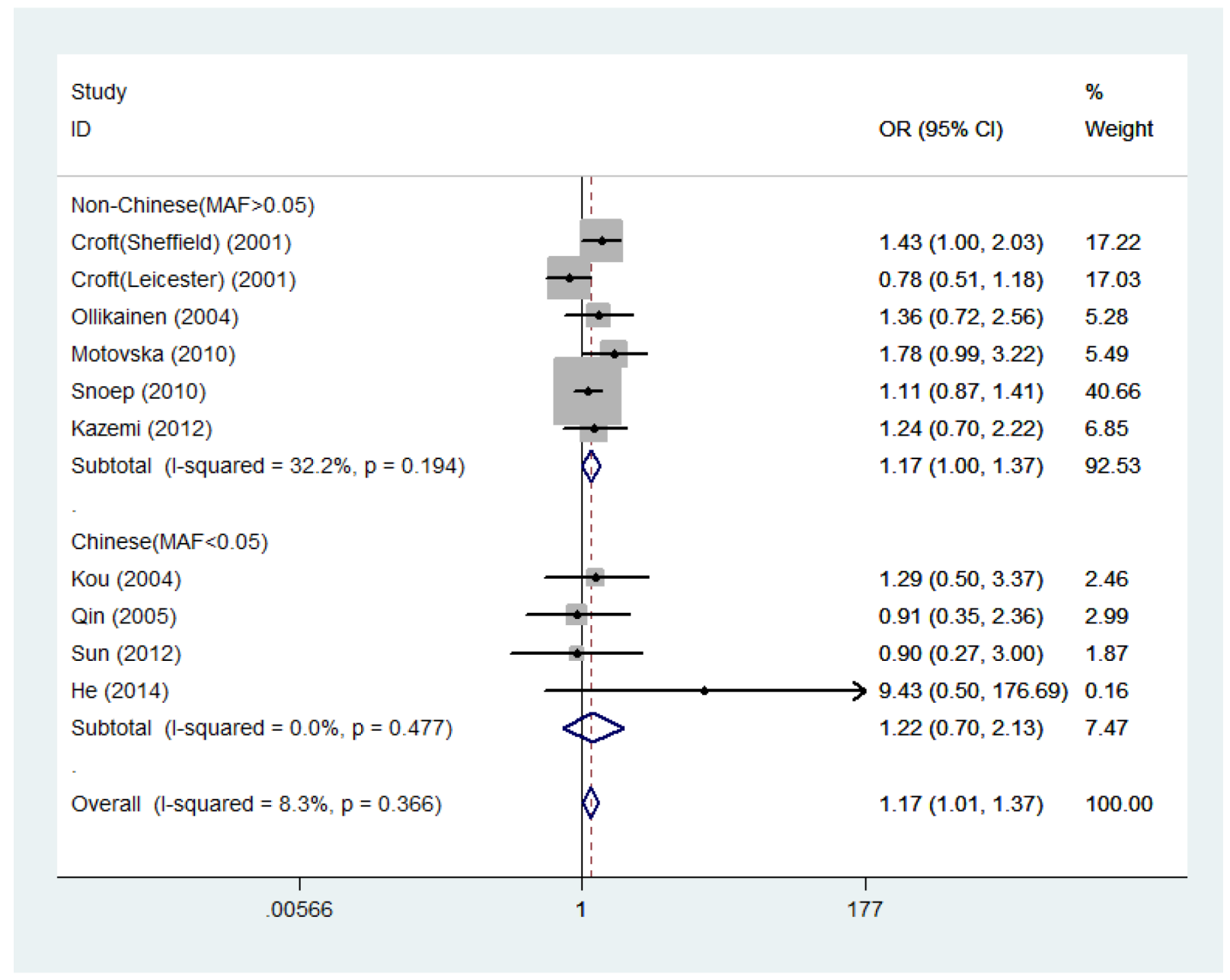

Figure 3: Forest plot for the dominant genetic model stratified by ethnicity (minor allele frequency of GP6 13254T $>$ C polymorphism) in studies with coronary artery disease patients. 
polymorphisms and CAD, published before May 2017 on the electronic databases PubMed, Embase, Chinese National Knowledge Infrastructure (CNKI), and Wanfang using the following search terms: (coronary artery disease or coronary heart disease atherosclerosis or myocardial infarction or myocardial infarct or heart attack or MI) and (polymorphism or single nucleotide polymorphism or SNP or variant or variation) and (glycoprotein VI or GP VI or GP6 or platelet membrane glycoprotein). No language restrictions were used. All eligible studies were retrieved, and cited references were carefully examined for additional potentially relevant studies.

The included articles in the meta-analysis should conform to the criteria as follows: (a) case-control
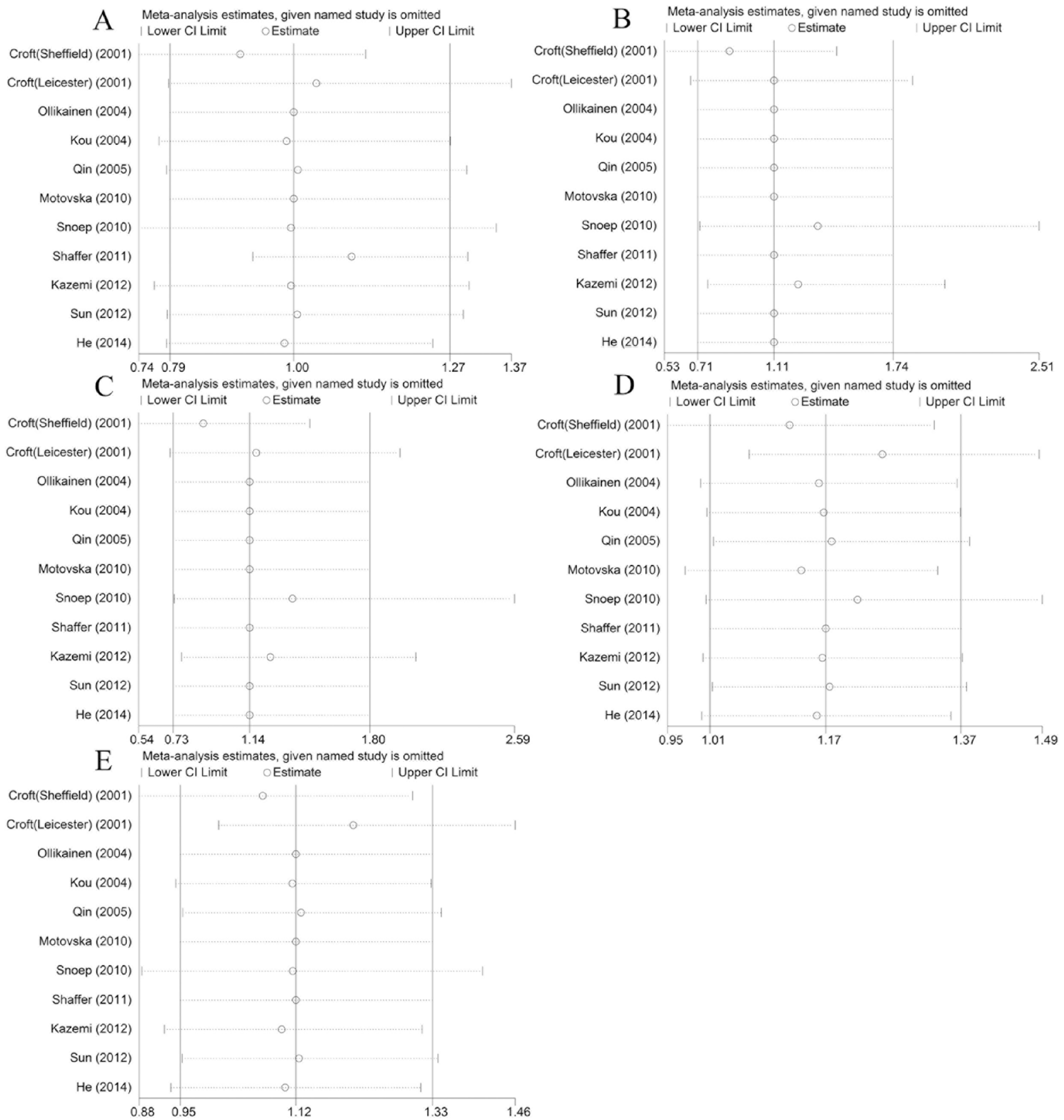

Figure 4: The sensitivity analysis of the pooled ORs and 95\% CIs for GP6 13254T $>$ C polymorphism under the allelic (A) recessive (B) homozygous (C) dominant (D) and heterozygous (E) genetic models. CI, confidence interval; OR, odds ratio. 
Table 3: Sensitivity analysis for the GP6 13254T>C genetic polymorphism under the dominant and heterozygous genetic models

\begin{tabular}{lcccccccc}
\hline \multirow{2}{*}{ First author } & \multirow{2}{*}{ Year } & \multicolumn{3}{c}{ Dominant genetic model } & & \multicolumn{2}{c}{ Heterozygous genetic model } \\
\cline { 3 - 4 } \cline { 7 - 8 } & & Estimate & \multicolumn{2}{c}{$\mathbf{9 5 \%}$ CI } & & Estimate & \multicolumn{2}{c}{$\mathbf{9 5 \%}$ CI } \\
\hline Croft (Sheffield) & 2001 & 1.12 & 0.95 & 1.33 & & 1.07 & 0.88 & 1.30 \\
Croft (Leicester) & 2001 & 1.26 & 1.06 & 1.48 & & 1.21 & 1.01 & 1.46 \\
Ollikainen & 2004 & 1.16 & 0.99 & 1.36 & & 1.12 & 0.95 & 1.33 \\
Kou & 2004 & 1.17 & 1.00 & 1.37 & & 1.12 & 0.94 & 1.33 \\
Qin & 2005 & 1.18 & 1.01 & 1.38 & & 1.13 & 0.95 & 1.34 \\
Motovska & 2010 & 1.14 & 0.97 & 1.34 & & 1.12 & 0.95 & 1.33 \\
Snoep & 2010 & 1.22 & 1.00 & 1.49 & & 1.12 & 0.89 & 1.41 \\
Shaffer & 2011 & 1.17 & 1.01 & 1.37 & & 1.12 & 0.95 & 1.33 \\
Kazemi & 2012 & 1.17 & 1.00 & 1.37 & & 1.10 & 0.92 & 1.32 \\
Sun & 2012 & 1.18 & 1.01 & 1.38 & & 1.13 & 0.95 & 1.34 \\
He & 2014 & 1.16 & 0.99 & 1.35 & & 1.11 & 0.93 & 1.31 \\
Combined & & 1.17 & 1.01 & 1.37 & & 1.12 & 0.95 & 1.33 \\
\hline
\end{tabular}

95\% CI, 95\% confidence interval.

design. (b) assessment of the association of GP6 genetic polymorphisms with CAD. c) data on the GP6 genotypes was available in both cases and controls. d) Genotypes in the control subjects should be in agreement with the Hardy-Weinberg equilibrium (HWE). Studies were excluded if any of the following applies: (a) reviews and repeated publications; (b) studies not meeting all of the inclusion criteria.

\section{Data extraction}

Data were independently extracted according to a standardized protocol by two investigators. The third one was to resolve the possible discrepancies between the two investigators. The similar data in different studies by the same author group were only adopted once. The following information was drawn out: first author's name, publication year, region, ethnicity, sample size, genotyping method and number of genotype in case and control group.

\section{Statistical analysis}

The odds ratio (OR) and their corresponding 95\% confidence interval (CI) were used to compare the association between GP6 $13254 \mathrm{~T}>\mathrm{C}$ polymorphism and CAD. The pooled ORs were assessed with allelic

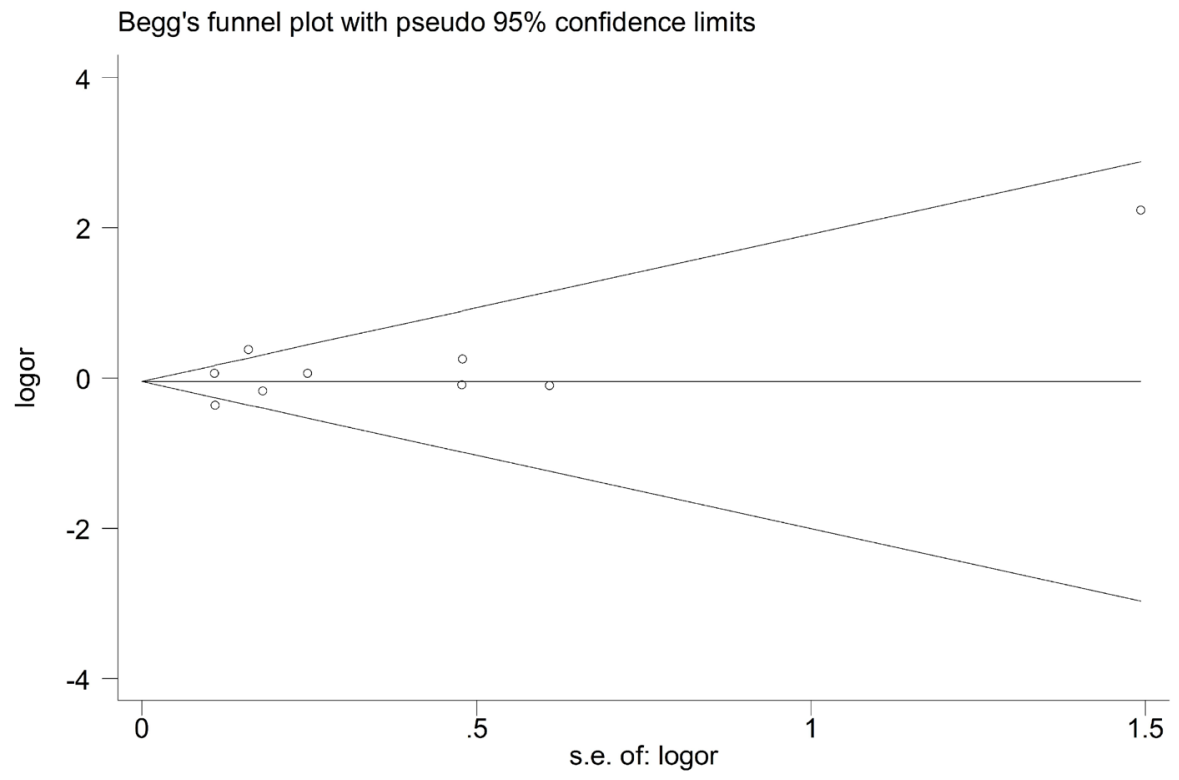

Figure 5: Begg's funnel plot for studies of the association between coronary artery disease and GP6 13254T $>$ C polymorphism under an allelic genetic model. 
Table 4: Egger's and Begg's test for the publication bias of GP6 13254T $>C$ genetic polymorphism

\begin{tabular}{lcc}
\hline Genetic Models & Egger's Test $\boldsymbol{p}$ Value & Begg's Test $\boldsymbol{p}$ Value \\
\hline Allelic genetic model & 0.369 & 0.754 \\
Dominant genetic model & 0.402 & 0.592 \\
Recessive genetic model & 0.614 & 0.734 \\
Homozygous genetic model & 0.596 & 0.734 \\
Heterozygous genetic model & 0.590 & 0.711 \\
\hline
\end{tabular}

model (C versus $\mathrm{T})$, homozygous model (CC versus TT), heterozygous model (TC versus TT), recessive model (CC versus TC/TT), dominant model (CC/TC versus TT). The $Z$ test was used to determine the pooled ORs with the significance set at $P<0.05$. The Chi-square test was used to assess the HWE in the control groups. The betweenstudy heterogeneity was determined by the $I^{2}$ statistic test, which is not inherently dependent on the number of studies for the meta-analysis [35]. If obvious heterogeneity existed among the individual studies $\left(I^{2}>50 \%\right)$, the randomeffects model would be used to calculate the pooled OR and its $95 \%$ CI [36]. If no heterogeneity is detected, the fixed-effects model using the Mantel-Haenszel method would be adopted for the meta-analysis [37]. Subgroup analysis according to the ethnicity or the MAF value was conducted to evaluate the association and explore the sources of between-study heterogeneity. Sensitivity analysis was performed to assess the effect of individual study on the combined results and evaluate the stability of results. The potential publication bias was detected by the Begg's funnel plot [38], and the funnel plot asymmetry was evaluated with the Egger's linear regression test [39]. The STATA 12.0 software (StataCorp, College Station, TX, USA) was used to perform all statistical analysis.

\section{ACKNOWLEDGMENTS AND FUNDING}

This work was supported by grants of the National Natural Scientific foundation of China (No. 81703623).

\section{CONFLICTS OF INTEREST}

The authors declare no competing financial interests.

\section{REFERENCES}

1. Wong ND. Epidemiological studies of CHD and the evolution of preventive cardiology. Nat Rev Cardiol. 2014; 11:276-289.

2. Girelli D, Piubelli C, Martinelli N, Corrocher R, Olivieri O. A decade of progress on the genetic basis of coronary artery disease. Practical insights for the internist. Eur J Intern Med. 2017; 41:10-17.

3. Mehta PK, Wei J, Wenger NK. Ischemic heart disease in women: a focus on risk factors. Trends Cardiovasc Med. $2015 ; 25: 140-151$.
4. Chen T, Wu B, Lin R. Association of apelin and apelin receptor with the risk of coronary artery disease: a metaanalysis of observational studies. Oncotarget. 2017; 8:57345-57355. https://doi.org/10.18632/oncotarget.17360.

5. Posadas-Sánchez R, Pérez-Hernández N, RodríguezPérez JM, Coral-Vázquez RM, Roque-Ramírez B, Llorente L, Lima G, Flores-Dominguez C, Villarreal-Molina T, PosadasRomero C, Vargas-Alarcón G. Interleukin-27 polymorphisms are associated with premature coronary artery disease and metabolic parameters in the Mexican population: the genetics of atherosclerotic disease (GEA) Mexican study. Oncotarget. 2017; 8:64459-64470. https://doi.org/10.18632/ oncotarget.16223.

6. Dai X, Wiernek S, Evans JP, Runge MS. Genetics of coronary artery disease and myocardial infarction. World $\mathrm{J}$ Cardiol. 2016; 8:1-23.

7. Edwards JJ, Gelb BD. Genetics of congenital heart disease. Curr Opin Cardiol. 2016; 31:235-241.

8. Morris DR, Moxon JV, Biros E, Krishna SM, Golledge J. Meta-analysis of the association between transforming growth factor-beta polymorphisms and complications of coronary heart disease. PLoS One. 2012; 7:e37878.

9. Luo JQ, Wen JG, Zhou HH, Chen XP, Zhang W. Endothelial nitric oxide synthase gene G894T polymorphism and myocardial infarction: a meta-analysis of 34 studies involving 21,068 subjects. PLoS One. 2014; 9:e87196.

10. Miao Q, Wang Q, Dong L, Wang Y, Tan Y, Zhang X. The expression of p66shc in peripheral blood monocytes is increased in patients with coronary heart disease and correlated with endothelium-dependent vasodilatation. Heart Vessels. 2015; 30:451-457.

11. Zhou JP, Bai YP, Hu XL, Kuang DB, Shi RZ, Xiong Y, Zhang W, Xia J, Chen BL, Yang TL, Chen XP. Association of the AGXT2 V140I polymorphism with risk for coronary heart disease in a Chinese population. J Atheroscler Thromb. 2014; 21:1022-1030.

12. Nieswandt B, Watson SP. Platelet-collagen interaction: is GPVI the central receptor? Blood. 2003; 102:449-461.

13. Clemetson JM, Polgar J, Magnenat E, Wells TN, Clemetson KJ. The platelet collagen receptor glycoprotein VI is a member of the immunoglobulin superfamily closely related to FcalphaR and the natural killer receptors. J Biol Chem. 1999; 274:29019-29024.

14. Jandrot-Perrus $M$, Busfield $\mathrm{S}$, Lagrue AH, Xiong $\mathrm{X}$, Debili N, Chickering T, Le Couedic JP, Goodearl A, Dussault B, Fraser C, Vainchenker W, Villeval JL. Cloning, 
characterization, and functional studies of human and mouse glycoprotein VI: a platelet-specific collagen receptor from the immunoglobulin superfamily. Blood. 2000; 96:1798-1807.

15. Jamasbi J, Megens RT, Bianchini M, Münch G, Ungerer M, Faussner A, Sherman S, Walker A, Goyal P, Jung S, Brandl R, Weber C, Lorenz R, et al. Differential Inhibition of Human Atherosclerotic Plaque-Induced Platelet Activation by Dimeric GPVI-Fc and Anti-GPVI Antibodies: Functional and Imaging Studies. J Am Coll Cardiol. 2015; 65:2404-2415.

16. Zahid M, Mangin P, Loyau S, Hechler B, Billiald P, Gachet C, Jandrot-Perrus M. The future of glycoprotein VI as an antithrombotic target. J Thromb Haemost. 2012; 10:2418-2427.

17. Ezumi Y, Uchiyama T, Takayama H. Molecular cloning, genomic structure, chromosomal localization, and alternative splice forms of the platelet collagen receptor glycoprotein VI. Biochem Biophys Res Commun. 2000; 277:27-36.

18. Croft SA, Samani NJ, Teare MD, Hampton KK, Steeds RP, Channer KS, Daly ME. Novel platelet membrane glycoprotein VI dimorphism is a risk factor for myocardial infarction. Circulation. 2001; 104:1459-1463.

19. Qin Q, Zhao BR, Mao YM, Cao RZ, Kou L, Li YL, Zhao FM, Hui RT. Association of matrix metalloproteinase-9 and platelet membrane glycoprotein VI polymorphisms with acute coronary syndrome. Chinese Journal of Cardiology. 2005; 33:622-626.

20. Yu ZQ, Dong NZ, Gao WQ, Bai X, Ruan CG. Study on T13254C polymorphism of the platelet membrane glycoprotein VI in Chinese Han population. Chinese Journal of Hematology. 2005; 26:140-143.

21. He JD, Tao L. A genetic association study for polymorphisms of platelet membrane glycoprotein and coronary artery disease. Journal of the American College of Cardiology. 2014; 64:C13.

22. Stroup DF, Berlin JA, Morton SC, Olkin I, Williamson GD, Rennie D, Moher D, Becker BJ, Sipe TA, Thacker SB. Meta-analysis of observational studies in epidemiology: a proposal for reporting. Meta-analysis Of Observational Studies in Epidemiology (MOOSE) group. JAMA. 2000; 283:2008-2012.

23. Ollikainen E, Mikkelsson J, Perola M, Penttilä A, Karhunen PJ. Platelet membrane collagen receptor glycoprotein VI polymorphism is associated with coronary thrombosis and fatal myocardial infarction in middle-aged men. Atherosclerosis. 2004; 176:95-99.

24. Sun YF, Cao J, Li XL, Fan L, Wang Q, Wang H, Zhang H, Yang L, Zhang F. Correlation of coronary heart disease with multiple genes, gene polymorphisms and multiple risk factors in old Chinese Han patients. Chinese Journal of Applied Physiology. 2012; 28:411-417.

25. Motovska Z, Kvasnicka J, Widimsky P, Petr R, Hajkova J, Bobcikova P, Osmancik P, Odvodyova D, Katina S. Platelet glycoprotein GP VI 13254C allele is an independent risk factor of premature myocardial infarction. Thrombosis Research. 2010; 125:e61-e64.

26. Snoep JD, Gaussem P, Eikenboom JC, Emmerich J, Zwaginga JJ, Holmes CE, Vos HL, de Groot PG, Herrington DM, Bray PF, Rosendaal FR, van der Bom JG. The minor allele of GP6 T13254C is associated with decreased platelet activation and a reduced risk of recurrent cardiovascular events and mortality: Results from the SMILE-Platelets project. Journal of Thrombosis and Haemostasis. 2010; 8:2377-2384.

27. Shaffer JR, Kammerer CM, Dorn J, Ferrell RE, Iacoviello L, Trevisan M, Donahue RP. Polymorphisms in the plateletspecific collagen receptor GP6 are associated with risk of nonfatal myocardial infarction in Caucasians. Nutrition, Metabolism and Cardiovascular Diseases. 2011; 21:546-552.

28. Kazemi A, Fatemi A, Givtaj N, Peighambari MM. Association of platelet collagen receptor polymorphisms with premature acute myocardial infarction. Blood Coagulation and Fibrinolysis. 2012; 23:527-531.

29. Kou L, Qin Q, Cao RZ, Zhao FM, Zhao BR. A Study on B $\beta$-Fibrinogen -148C/T Polymorphism and Platelet Membrane Glycoprotein VI T13254C Polymorphism of Coronary Heart Disease. Molecular Cardiology of China. 2004; 4:334-338.

30. Takagi S, Iwai N, Baba S, Mannami T, Ono K, Tanaka C, Miyata T, Miyazaki S, Nonogi H, Goto Y. A GPVI polymorphism is a risk factor for myocardial infarction in Japanese. Atherosclerosis. 2002; 165:397-398.

31. Joutsi-Korhonen L, Smethurst PA, Rankin A, Gray E, IJsseldijk M, Onley CM, Watkins NA, Williamson LM, Goodall AH, de Groot PG, Farndale RW, Ouwehand WH. The low-frequency allele of the platelet collagen signaling receptor glycoprotein VI is associated with reduced functional responses and expression. Blood. 2003; 101:4372-4379.

32. Best D, Senis YA, Jarvis GE, Eagleton HJ, Roberts DJ, Saito T, Jung SM, Moroi M, Harrison P, Green FR, Watson SP. GPVI levels in platelets: relationship to platelet function at high shear. Blood. 2003; 102:2811-2818.

33. Trifiro E, Williams SA, Cheli Y, Furihata K, Pulcinelli FM, Nugent DJ, Kunicki TJ. The low-frequency isoform of platelet glycoprotein VIb attenuates ligand-mediated signal transduction but not receptor expression or ligand binding. Blood. 2009; 114:1893-1899.

34. Liu XC, Wang Z. The significance of CHI3L1, GP6 gene polymorphism detection in coronary artery disease of Han population. Hebei Medical University Master's Thesi 2016.

35. Higgins JP, Thompson SG, Deeks JJ, Altman DG. Measuring inconsistency in meta-analyses. BMJ. 2003; 327:557-560.

36. DerSimonian R, Laird N. Meta-analysis in clinical trials. Control Clin Trials. 1986; 7:177-188.

37. Mantel N, Haenszel W. Statistical aspects of the analysis of data from retrospective studies of disease. J Natl Cancer Inst. 1959; 22:719-748. 
38. Begg CB, Mazumdar M. Operating characteristics of a rank correlation test for publication bias. Biometrics. 1994; 50:1088-1101.
39. Egger M, Davey Smith G, Schneider M, Minder C. Bias in meta-analysis detected by a simple, graphical test. BMJ. 1997; 315:629-634. 